\title{
TOWARDS ANALYSIS OF FOOT MOTION USING DYNAMIC 3D SURFACE SCANNING
}

\author{
Inge Van den Herrewegen (1), Kris Cuppens (1), Mario Broeckx (1), Helga Vertommen \\ (1), Marc Mertens (1), Louis Peeraer (1),(2)
1. KHK-Mobilab, Belgium; 2.KU Leuven -Faculty of Kinesiology and Rehabilitation Science, Belgium

\section{Introduction}

Until now foot motion is mostly studied by markerbased 3D analysis. However, a recently developed dynamic 3D scanner is able to scan the entire foot surface at $49 \mathrm{~Hz}$ (Vialux). This creates a variety of possibilities for analysis of foot motion during gait. The aim of this study is the extraction of a segmented foot model from a dynamic 3D scan. Such a foot model would enable a motion analysis without the need to predefine segments, with data from quick and non-invasive measurements.

\section{Methods}

The dynamic 3D foot scan system comprises 5 scanners, and foot analysis software developed by sportmedicin Tübingen [T. Schmeltzpfenning, 2009]. Scanners are mounted around a glass force plate in such a way that the entire foot surface is scanned including the plantar surface of the foot (figure 1). This outputs a dynamic point cloud of the geometry of the foot during the whole of the stance phase of gait.

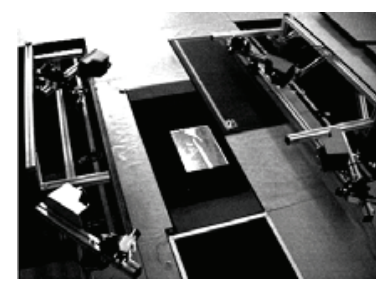

Figure 1: Dynamic 3D scanning setup

To extract a segmented foot model from the 3D images, an algorithm identifying rigid segments in the dynamic point cloud has to be developed. A part of the foot is considered 'rigid' when all of its points move alike, so it can be rigid during a certain motion, but may split into more segments in another situation. A footstep typically combines several movements, and hence needs to be segmented frame by frame.

To define rigid segments in 2 subsequent 3D frames, the motion between those frames is calculated using a Brox optical flow algorithm. This is a mathematical technique to calculate the motion vector field between 2 subsequent frames. This vector field shows which points move alike, and makes segment and rotation axis calculation possible (figure 2). a)
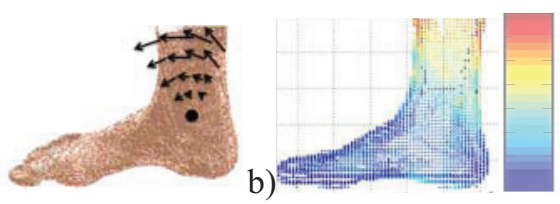

Figure 2: simplified example of a vector field, (a) direction and (b) norm with colour bar. Rotation axis \& segment can be extracted from these vectors.

\section{Results}

As a proof of concept, foot motion vector fields in three stages of a footstep are calculated (figure $3 a$ ). For each of these stadia, the moving segments and their rotation axes can be calculated. The accuracy of these calculations is verified on the scan of an artificial foot with known axes and real rigid segments (figure 3b). Segments are identified correctly when averaged over 4 subsequent frames. Axis direction is detected with an accuracy of $11^{\circ}$.

a)

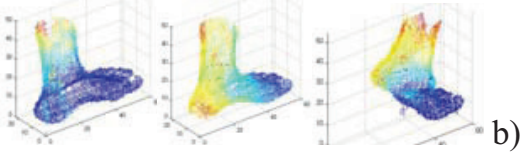

b)

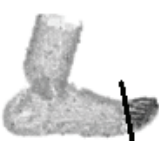

Figure 3: a) foot motion vector fields (norm) during midstance, heel off and toe off. b) artificial foot with detected toe rotation axis and toe segment

\section{Discussion}

Dynamic 3D scanning is a novel alternative to analyse the foot, yet still expensive. To retrieve meaningful information, extraction of a segmented foot model based on the flow field has shown to be promising. The axis detection can still be improved, as these initial calculations were performed with a coarse quantization, and great improvement is to be expected in refined calculations. Future work will apply improved segmentation and axis calculation algorithms on scanned human footsteps.

\section{Acknowledgements}

Many thanks to prof. J. Barron for his support using the optical flow implementation.

\section{References}

T. Schmeltzpfenning et al, Footwear Science, 1(S1): 28-30, 2009. 